\title{
Clinical outcome of neurological patients with COVID-19: the impact of healthcare organization improvement between waves
}

\author{
Viviana Cristillo ${ }^{1}$ (D) Andrea Pilotto ${ }^{1}$ - Alberto Benussi ${ }^{1} \cdot$ Ilenia Libri ${ }^{1} \cdot$ Marcello Giunta ${ }^{1}$. Andrea Morotti ${ }^{1}$. \\ Stefano Gipponi ${ }^{1} \cdot$ Martina Locatelli $^{1}$ - Stefano Cotti Piccinelli ${ }^{1}$. Valentina Mazzoleni ${ }^{1} \cdot$ Francesca Schiano di Cola $^{1}$. \\ Stefano Masciocchi ${ }^{1} \cdot$ Debora Pezzini $^{1}$ - Andrea Scalvini ${ }^{1}$ - Enrico Premi ${ }^{2}$ - Elisabetta Cottini ${ }^{1} \cdot$ Massimo Gamba $^{2}$. \\ Mauro Magoni ${ }^{2} \cdot$ Marco Maria Fontanella $^{3} \cdot$ Alessandro Padovani $^{1}$
}

Received: 3 November 2021 / Accepted: 6 February 2022 / Published online: 17 February 2022

(c) The Author(s) 2022

\begin{abstract}
Objective The aim of this study is to evaluate the differences in clinical presentations and the impact of healthcare organization on outcomes of neurological COVID-19 patients admitted during the first and second pandemic waves.

Methods In this single-center cohort study, we included all patients with SARS-CoV-2 infection admitted to a Neuro-COVID Unit. Demographic, clinical, and laboratory data were compared between patients admitted during the first and second waves of the COVID-19 pandemic.

Results Two hundred twenty-three patients were included, of whom 112 and 111 were hospitalized during the first and second pandemic waves, respectively. Patients admitted during the second wave were younger and exhibited pulmonary COVID-19 severity, resulting in less oxygen support $(n=41,36.9 \%$ vs $n=79,70.5 \%, p<0.001)$ and lower mortality rates $(14.4 \%$ vs $31.3 \%, p=0.004)$. The different healthcare strategies and early steroid treatment emerged as significant predictors of mortality independently from age, pre-morbid conditions and COVID-19 severity in Cox regression analyses.

Conclusions Differences in healthcare strategies during the second phase of the COVID-19 pandemic probably explain the differences in clinical outcomes independently of disease severity, underlying the importance of standardized early management of neurological patients with SARS-CoV-2 infection.
\end{abstract}

Keywords COVID-19 $\cdot$ Neurological disease $\cdot$ Outcomes $\cdot$ Steroid therapy $\cdot$ Mortality

\section{Introduction}

Coronavirus disease-19 (COVID-19), associated with the severe acute respiratory syndrome coronavirus 2 (SARSCoV-2) has become a global pandemic, giving rise to a serious health burden globally. Many countries worldwide experienced a two-wave pattern of COVID-19 spreading

Viviana Cristillo

viviana.cristillo@gmail.com

1 Department of Clinical and Experimental Sciences, Neurology Unit, University of Brescia, P. le Spedali Civili 1, 25123 Brescia, Italy

2 Stroke Unit, Azienda Socio Sanitaria Territoriale Spedali Civili, Spedali Civili Hospital, Brescia, Italy

3 Neurosurgery Unit, Department of Medical and Surgical Specialties, Radiological Sciences and Public Health, University of Brescia, Spedali Civili di Brescia, Brescia, Italy during the pandemic, with a first wave during spring 2020 [1] followed by the second wave starting in late summer 2020 persisting until the spring of 2021. Neurological symptoms and syndromes concomitant SARS-coV-2 infection have been associated with increased risk of mortality and poor outcome in independent case series [1-4]. Recent data from general COVID units suggested that the patients hospitalized during the first and second waves of the COVID19 pandemic differed for age range, severity of the disease, COVID-19 treatment strategies adopted, and outcomes [5] but no data specifically evaluating neurological patients are still available.

In this work, we aimed to evaluate the impact of different healthcare strategies on final outcomes, by comparing clinical and laboratory characteristics of hospitalized COVID-19 neurological patients during the first and second waves of the pandemic in a single territory hub for neuro-COVID patients. 


\section{Methods}

\section{Study design and participants}

This cohort study included adult inpatients ( $\geq 18$ years old) with SARS-CoV2 infection admitted at the NeuroCOVID Unit of the ASST Spedali Civili Hospital, Brescia, for neurological diseases from February 21 to June 5, 2020 (first wave) and from November 1 to April 30, 2021 (second wave). In both periods, the hospital was selected as the hub for strokes. SARS-CoV-2 infection was confirmed by RT-PCR in nasopharyngeal/oropharyngeal swabs or bronchoalveolar lavage. The study received approval from the local ethics committee of the ASST Spedali Civili Hospital, Brescia (NP 4067, approved 08.05. 2020). Pre-morbid conditions were recorded at admission using the cumulative Illness rating scale (CIRS) and the premorbid modified Rankin scale (mRS). Hospitalization data included the severity of COVID-19 disease, expressed by the Brescia-COVID Respiratory Severity Scale (BCRSS) [6] and the quick Sequential Organ Failure Assessment (qSOFA) score.

Steroid treatment with methylprednisolone $1 \mathrm{~g} /$ day for 5 days was defined as high-dose treatment (HDS), whereas dexamethasone $6 \mathrm{mg} /$ day was defined as standard-dose treatment (SDT). During the second wave, the health organisation system changed adopting (a) different referral system from family doctors for patients at higher risk of deterioration; (b) standardization of patients profiling using neurological, comorbid, and frailty measures; (c) standardization of management of neurological patients in COVID-19 including specific internal guidelines for stroke, encephalitis, delirium, seizures, and headache; (d) multidisciplinary team of clinicians including neurologist, internal medicine, and infectious disease specialists in the unit; (e) early use of steroid and heparin according to updated COVID-19 guidelines [7]; (f) larger use of non-invasive ventilation in non-ICU units; and $(\mathrm{g})$ early screening for ICU need.

\section{Statistical analysis}

Continuous and categorical variables are reported as mean values \pm standard deviation and $n(\%)$ respectively. Differences between patients during the two waves were compared by $t$-test or Fisher's exact test where appropriate. Linear regression models adjusted for the effect of age, COVID-19 severity, comorbidities, and baseline $\mathrm{mRS}$ evaluated the impact on different waves on $\mathrm{mRS}$ at discharge. The Cox regression model based on previous findings [1] was implemented in order to investigate the combined effect of predictors of mortality, namely age, qSOFA scores, BCRSS, platelet count, first vs. second wave, steroid treatment, and time from symptoms onset to admission. A two-sided $p$-value $<0.05$ was considered significant; data analyses were carried out using SPSS software (version 21.0).

\section{Results}

Two hundred twenty-three COVID-19 patients were hospitalized in the Neuro-COVID Unit of the ASST Spedali Civili di Brescia Hospital, of whom 112 were admitted from February 21 to June 5, 2020, and 111 were hospitalized from November 1 to April 30, 2021.

Demographic, clinical, and laboratory characteristics of included patients are reported in Table 1.

Patients admitted during the second wave were younger (years $62.9 \pm 18.9$ vs. $72.6 \pm 12.1, p<0.001$ ), exhibited a lower comorbidity severity index $(1.21 \pm 0.2$ vs. $1.28 \pm 0.2$, $p=0.026$ ), less severe pulmonary disease, expressed by lower qSOFA score $(0.48 \pm 0.7$ vs. $0.87 \pm 0.7, p<0.001)$, and lower BCRSS $(0.50 \pm 0.8$ vs. $1.24 \pm 0.97, p<0.001)$ at admission compared to patients hospitalized during the first outbreak.

COVID-19 patients admitted during the first wave showed higher blood inflammatory parameters, chest X-ray scores (Table 1) and use of high flow oxygenation ( $n=79,70.5 \%$, $n=41,36.9 \%$ vs. $p<0.001$ ) compared to patients admitted during the second outbreak.

The specific neurological diagnosis exhibited a different distribution within the two time-periods (Fig. 1) characterized by a significant reduction in stroke rates during the second wave ( $n=54,48.2 \%$ vs. $n=25,22.5 \%, p<0.001)$. Patients with moderate to severe respiratory disease exhibited a shorter time from symptom onset to hospitalization in the second wave compared to the first pandemic phases (days $0.40 \pm 0.74$ vs $2.79 \pm 7.5, p=0.037$ ). During the second wave, patients showed lower mortality rates after adjusting for age and COVID-19 severity ( $n=32,38.6 \%$ vs. $n=19$, $17.1 \%, p=0.009$ ), and better clinical outcomes adjusting for baseline status (mRS at discharge $2.40 \pm 1.6$ vs $1.81 \pm 1.7$, $p=0.019$ ).

The Cox regression model identified age $(p=0.001)$, COVID-19 severity (i.e., BCRSS, $p<0.001$ ), pre-morbid comorbidity (i.e., CIRS, $p=0.028$ ), and the different time period (i.e., waves, $p=0.012$ ) as independent significant predictors of mortality in hospitalized patients. Standard steroid treatment was adopted in $4.7 \%$ and $48.1 \%$ of patients admitted during the first and second waves, respectively $(p<0.001)$. Specific Cox regression analyses revealed steroid treatment as an independent predictor of survival (ExpB 2.084, IC 1.072-4.050, $p=0.007$ ) after adjusting for age, 
Table 1 Demographic, clinical, laboratory characteristics of patients according to first, and second pandemic waves

\begin{tabular}{|c|c|c|c|c|}
\hline & $\begin{array}{l}\text { Neuro-COVID } \\
\text { Total }(n=223)\end{array}$ & $\begin{array}{l}\text { Neuro-COVID } \\
1^{\circ} \text { wave }(n=112)\end{array}$ & $\begin{array}{l}\text { Neuro-COVID } \\
2^{\circ} \text { wave }(n=111)\end{array}$ & $* p$ value \\
\hline \multicolumn{5}{|l|}{ Clinical and demographics features } \\
\hline Age, years & $67.74 \pm 16.5$ & $72.6 \pm 12.1$ & $62.9 \pm 18.9$ & $<0.001$ \\
\hline Sex, female & $107(48 \%)$ & $53(47.3 \%)$ & $54(48.6 \%)$ & 0.843 \\
\hline Hospital length of stay, days & $11.6 \pm 10.1$ & $9.75 \pm 7.9$ & $13.55 \pm 11.6$ & 0.005 \\
\hline Time to admission from onset, days ${ }^{\circ}$ & $1.97 \pm 4.75$ & $2.79 \pm 7.5$ & $0.40 \pm 0.74$ & $\mathbf{0 . 0 3 7}$ \\
\hline qSOFA score, mean & $0.67 \pm 0.7$ & $0.87 \pm 0.7$ & $0.48 \pm 0.7$ & $<0.001$ \\
\hline BCRSS, mean & $0.87 \pm 0.96$ & $1.24 \pm 0.97$ & $0.50 \pm 0.8$ & $<0.001$ \\
\hline mRS pre-admission & $1.40 \pm 1.4$ & $1.22 \pm 1.2$ & $1.59 \pm 1.6$ & 0.053 \\
\hline CIRS pre-admission & $1.24 \pm 0.2$ & $1.28 \pm 0.2$ & $1.21 \pm 0.2$ & 0.026 \\
\hline Lymphocytes & $1.36 \pm 0.8$ & $1.21 \pm 0.6$ & $1.51 \pm 0.9$ & 0.006 \\
\hline C-reactive protein (mg/L) & $41.2 \pm 61.7$ & $50.5 \pm 67.4$ & $31.96 \pm 54.2$ & 0.027 \\
\hline Creatine kinase (U/L) & $219.4 \pm 554.5$ & $276.38 \pm 755.6$ & $165.6 \pm 235.7$ & 0.159 \\
\hline D-dimer (mg/L) & $1742.4 \pm 2986.2$ & $1385.6 \pm 1683.1$ & $2032.5 \pm 3707.9$ & 0.109 \\
\hline \multicolumn{5}{|l|}{ Therapy } \\
\hline Oxygen therapy, $n(\%)$ & $120(53.8 \%)$ & $79(70.5 \%)$ & $41(36.9 \%)$ & $<0.001$ \\
\hline High-dose treatment, $n(\%)$ & $10(4.5 \%)$ & $4(3.6 \%)$ & $6(5.4 \%)$ & 0.479 \\
\hline Standard-dose treatment, $n(\%)$ & $55(24.7 \%)$ & $5(4.7 \%)$ & $50(48.1 \%)$ & $<0.001$ \\
\hline \multicolumn{5}{|l|}{ Outcomes measures } \\
\hline In hospital mortality, $n(\%)$ & $51(22.9 \%)$ & $35(31.3 \%)$ & $16(14.4 \%)$ & 0.004 \\
\hline $\mathrm{mRS}$ at discharge (death included) & $2.98 \pm 2.2$ & $3.53 \pm 2.1$ & $2.41 \pm 2.1$ & $<0.001$ \\
\hline mRS at discharge (deaths excluded) & $2.08 \pm 1.7$ & $2.40 \pm 1.6$ & $1.81 \pm 1.7$ & 0.019 \\
\hline
\end{tabular}

${ }^{*} p$ values were calculated by $t$-test or Fisher's exact test, as appropriate $(p<0.05)$; ${ }^{\circ}$ patients with BCRSS $\geq 2$; abbreviations: BCRSS, BresciaCOVID Respiratory Severity Scale; CIRS, Cumulative Illness Rating Scale; GBS, Guillain-Barrè syndrome; mRS, modified Rankin scale; qSOFA, quick sequential organ failure assessment

BCRSS, CIRS, and time period-in the global sample (Supplementary Fig. 1).

\section{Discussion}

The study showed that patients admitted during the second wave of the COVID-19 pandemic were younger, exhibited lower pulmonary severity, a different distribution of neurological diagnosis, lower mortality rates, and better neurological outcomes compared to patients admitted during the first wave. The different healthcare strategies adopted during the two phases of the pandemic and the modulation with steroid treatments appeared to be independent predictors of mortality in addition to age, pre-morbid conditions, and COVID-19 severity in the cohort.

This study included 223 consecutive COVID-19 patients hospitalized for neurological disorders admitted during two different peaks of the pandemic in Italy in a tertiary hub for Neuro-COVID patient. During the first phases of the pandemic, we observed a higher prevalence of cerebrovascular diseases, representing more than half of patients evaluated in the emergency room [8]. During the second phase of the pandemic, conversely, we observed a slightly higher prevalence of patients hospitalized for encephalopathies or headaches, whereas cerebrovascular events decrease to about a third of admitted patients. These differences might be due to younger age, lower comorbidity status, and lower severity of COVID-19 observed during the second wave, as severe SARS-Cov-2 infection with prominent systemic response has been claimed to be associated with increased risk of stroke [2-4]. The Cox regression model identified the severity of pulmonary disease, age, and pre-morbid conditions as the most important predictors of mortality, with a strong difference in mortality rates between first and second waves $(38 \%$ vs $17 \%$ ). On the one hand, the general decrease of severity of COVID-19 disease during the second wave-reported in Europe and USA - might largely explain the reduction of mortality and medical complications observed [9-12]. On the other hand, the management of COVID-19 patients consistently improved during the second pandemic wave both at primary care and at the hospital level [13]. The total number of COVID-19 dedicated units and beds were increased along with both the non-invasive ventilation and patients were hospitalized earlier and with milder symptoms [10]. Second, the lessons learned from the first phases of the pandemic 
Fig. 1 Neurological diagnosis distribution during the first and second pandemic waves. Abbreviations: GBS, Guillain-Barrè syndrome; ICH, intracerebral hemorrhage, SAH, subarachnoid hemorrhage; TIA: transient ischemic attack

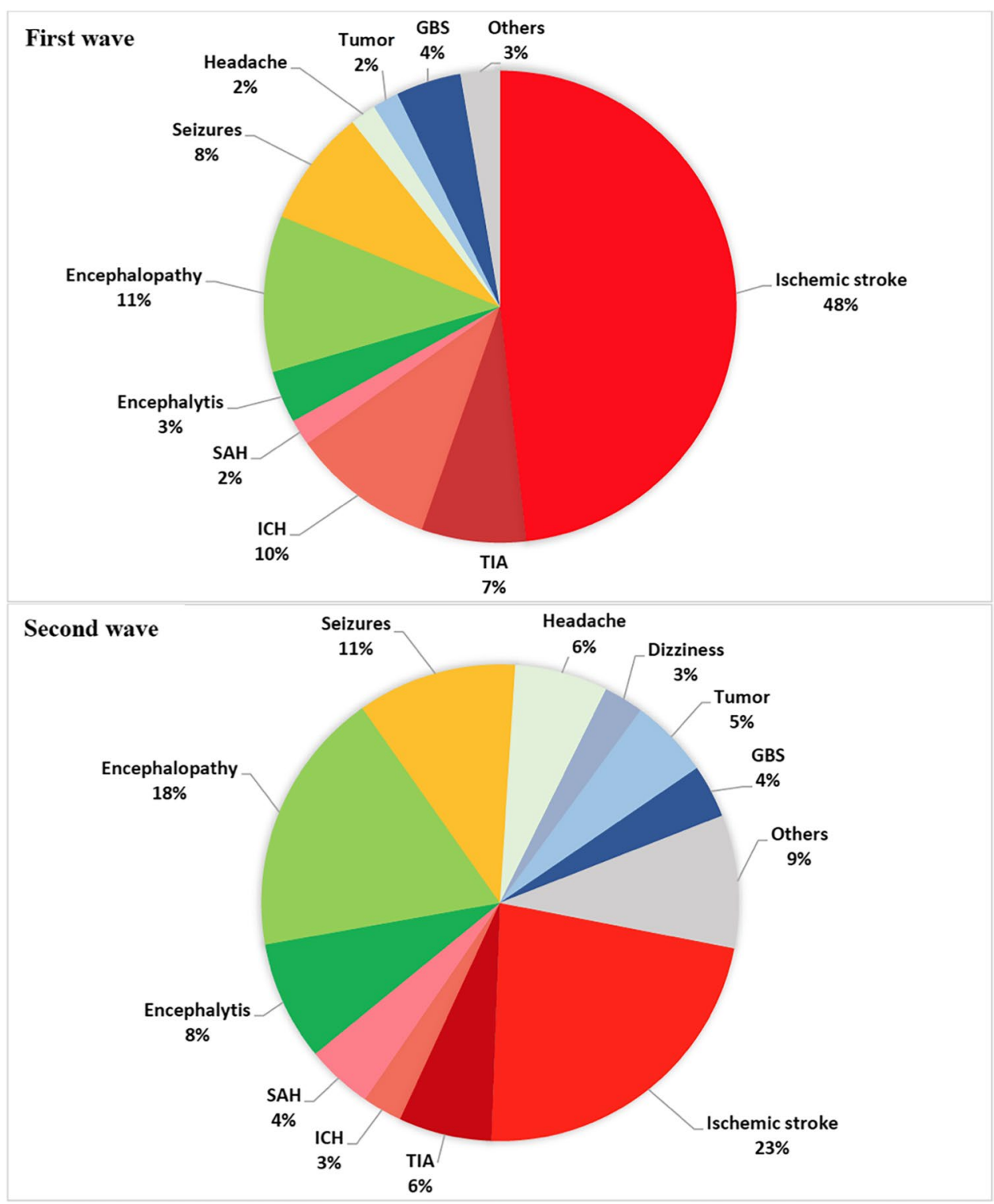

allowed the development of standardized procedures and guidelines for the management of patients with moderate and severe COVID-19 disease, thus strongly improving the care of patients since the early stages. Third, the primary care doctors were directly involved in the initial management of patients with COVID-19 infection, thus increasing the referral to specific COVID-19 units when needed [11]. Dexamethasone and remdesivir substituted hydroxychloroquine and lopinavir, and anticoagulation therapy was promptly administrated since the first days of admission [14]. Indeed, the time interval since onset appeared to improve survival and outcome independently from the severity of the disease. Furthermore, the known increased inflammatory response to the viral infection might be an important modulator of incidence and severity of related CNS disorders $[2-4,15]$ in addition to systemic complications. This fits with the results of our study, indicating immunomodulatory treatment as an independent predictor of mortality - in addition to the time of hospitalization and severity of the disease.

We acknowledge that this work entails some limitations, as this is a monocentric study with a relatively small sample size and we could not exclude that some patients with COVID-19 disease and neurological symptoms or syndromes did escape the referral, especially for mild cases not requiring hospitalization. Moreover, this is a retrospective study, and treatments' effects are evaluated by an observational approach.

Nevertheless, this is the first study evaluating the differences between neurological COVID-19 patients during the two pandemic waves. Findings showed that different management strategies adopted and the lessons learned by health workers from the first pandemic phases largely explain the 
improvement in final outcomes observed independently from the reduction of severity of SARS-CoV-2 infection. Larger ongoing multicenter studies are warranted to confirm and extend these findings in order to understand the future global impact of healthcare system organization, immunomodulatory treatments, and the large use of vaccination on the outcome of neurological patients with COVID-19 disease.

Supplementary Information The online version contains supplementary material available at https://doi.org/10.1007/s10072-022-05946-8.

\section{Declarations}

Competing interest V. Cristillo, A. Benussi, I. Libri, M. Giunta, A. Morotti, S. Gipponi, M. Locatelli, S. Cotti Piccinelli, V. Mazzoleni, F. Schiano di Cola, S. Masciocchi, D. Pezzini, A. Scalvini, E. Premi, E. Cottini, M. Gamba, M. Magoni, M. M. Fontanella report no disclosures relevant to the manuscript. Andrea Pilotto received speaker honoraria and travel grants from AbbVie Pharmaceuticals BioMarin Pharmaceutical, Chiesi Pharmaceuticals, Nutricia Pharmaceuticals, UCB Pharma, and Zambon Pharmaceuticals. Alessandro Padovani is consultant and served on the scientific advisory board of GE Healthcare, Eli-Lilly, and Actelion Ltd. Pharmaceuticals; and received speaker honoraria from Nutricia, PIAM, Lansgstone Technology, GE Healthcare, Lilly, UCB Pharma, and Chiesi Pharmaceuticals.

Ethical approval The Neuro-COVID Next study was approved by the local ethics committee of ASST Spedali Civili di Brescia Hospital (NP 4067, approved 08.05.2020).

Open Access This article is licensed under a Creative Commons Attribution 4.0 International License, which permits use, sharing, adaptation, distribution and reproduction in any medium or format, as long as you give appropriate credit to the original author(s) and the source, provide a link to the Creative Commons licence, and indicate if changes were made. The images or other third party material in this article are included in the article's Creative Commons licence, unless indicated otherwise in a credit line to the material. If material is not included in the article's Creative Commons licence and your intended use is not permitted by statutory regulation or exceeds the permitted use, you will need to obtain permission directly from the copyright holder. To view a copy of this licence, visit http://creativecommons.org/licenses/by/4.0/.

\section{References}

1. Benussi A, Pilotto A, Premi E et al (2020) Clinical characteristics and outcomes of inpatients with neurologic disease and COVID19 in Brescia, Lombardy, Italy. Neurology 95(7):e910-e920. https://doi.org/10.1212/WNL.0000000000009848

2. Ellul MA, Benjamin L, Singh B et al (2020) Neurological associations of COVID-19. Lancet Neurol 19:767-783
3. Paterson RW, Brown RL, Benjamin L et al (2020) The emerging spectrum of COVID-19 neurology: clinical, radiological and laboratory findings. Brain 143:3104-3120

4. Garcia-Azorin D, Martinez-Pias E, Trigo J et al (2020) Neurological comorbidity is a predictor of death in Covid-19 disease: a cohort study on 576 patients. Front Neurol 11:781. https://doi. org/10.3389/fneur.2020.00781

5. Iftimie S, Lopez-Azcona AF, Vallverdu I et al (2021) First and second waves of coronavirus disease-19: a comparative study in hospitalized patients in Reus, Spain. PLoS ONE 16(3):e0248029. https://doi.org/10.1371/journal.pone.0248029

6. Duca A, Piva S, Foca E et al (2020) Calculated decisions: BresciaCOVID respiratory severity scale (BCRSS)/algorithm. Emerg Med Pract 22(5 Suppl):CD1-CD2

7. Group RC, Horby P, Lim WS et al (2021) Dexamethasone in hospitalized patients with Covid-19. N Engl J Med 384(8):693-704. https://doi.org/10.1056/NEJMoa2021436

8. Pilotto A, Benussi A, Libri I et al (2021) COVID-19 impact on consecutive neurological patients admitted to the emergency department. J Neurol Neurosurg Psychiatry 92(2):218-220. https://doi.org/10.1136/jnnp-2020-323929

9. Chirico F, Nucera G, Szarpak L (2021) COVID-19 mortality in Italy: the first wave was more severe and deadly, but only in Lombardy region. J Infect 83(1):e16. https://doi.org/10.1016/j. jinf.2021.05.006[publishedOnlineFirst:2021/05/17]

10. Saito S, Asai Y, Matsunaga N et al (2021) First and second COVID-19 waves in Japan: a comparison of disease severity and characteristics. J Infect 82:84-123

11. Bongiovanni M, Arienti R, Bini F et al (2021) Differences between the waves in Northern Italy: How the characteristics and the outcome of COVID-19 infected patients admitted to the emergency room have changed. J Infect 83(1):e32-e33. https://doi.org/10. 1016/j.jinf.2021.04.024

12. Iftimie S, Lopez-Azcona AF, Vallverdu I et al (2021) First and second waves of coronavirus disease-19: a comparative study in hospitalized patients in Reus, Spain. PLoS One 16:e0248029

13. Garcia-Azorin D, Seeher KM, Newton CR et al (2021) Disruptions of neurological services, its causes and mitigation strategies during COVID-19: a global review. J Neurol. https://doi.org/10. 1007/s00415-021-10588-5

14. Lopes RD, de Barros ESPGM, Furtado RHM et al (2021) Therapeutic versus prophylactic anticoagulation for patients admitted to hospital with COVID-19 and elevated D-dimer concentration (ACTION): an open-label, multicentre, randomised, controlled trial. Lancet 397:2253-2263

15. Solomon T (2021) Neurological infection with SARS-CoV-2 - the story so far. Nat Rev Neurol 17(2):65-66. https://doi.org/10.1038/ s41582-020-00453-w

Publisher's note Springer Nature remains neutral with regard to jurisdictional claims in published maps and institutional affiliations. 